\title{
Factors influencing patient decision making in Urogynaecology: You are what you know
}

\author{
Kaven Baessler ${ }^{1}$ - Diaa E. E. Rizk ${ }^{2}$ \\ Received: 7 March 2020 / Accepted: 9 March 2020/Published online: 28 March 2020 \\ (C) The International Urogynecological Association 2020
}

In a recent professional conversation, a gynecological oncologist colleague exclaimed "You poor urogynaecologists! You do not know which single operation is the best because there are too many options so patients have to be counseled for ages. After your operations, recovery of function does not necessarily follow restored anatomy so women become frequently disappointed, even more so if they have a postoperative complication for a non-life-threatening condition. In contrast, my patients are simply happy if they survive after surgery". Although this seems to be the perception of our subspecialty among the gynecological community, we usually consider it a privilege having different conservative and surgical options for the management of pelvic floor disorders that we can offer to our patients. The privilege is, however, challenging for several reasons.

Over the last decade, we have learned what women with pelvic floor problems know, want and expect. We established patient-related outcome measures and later developed guidelines to include quality-of-life assessment in reporting treatment outcomes [1]. We already knew that many incontinent women do not seek help because they considered this a consequence of normal aging [2,3]. When we studied their care-seeking behavior, the influence of social, cultural, ethnic and religious factors was appreciated [4]. The majority of women perceived pelvic floor disorders as an intimate medical problem and therefore preferred to consult female health care providers $[4,5]$ without involving their partners or other family members [2]. In particular, patients usually preferred

Kaven Baessler

kaven.baessler@franziskus-berlin.de

1 Pelvic Floor Centre, Franziskus and St. Joseph Hospitals, Berlin, Budapester Str. 15-19, 10787 Berlin, Germany

2 Department of Obstetrics and Gynecology, College of Medicine and Medical Sciences, Arabian Gulf University, P.O. Box 26,

Manama ,671, Bahrain to receive care by a female urogynaecologist aged between 45 and 60 years with more than 10 years of experience [5]. Women with pelvic floor disorders were also more likely to seek care when the adverse impact on the quality of life was greater [2]. Concerns of affected women varied from worrying about body image like appearance and smell to improving sexual function, reducing pad use and becoming more physically active [6]. Research on patients goal achievement of treatment highlighted that many women preferred to be dry but would not mind if the incontinence only improves to be able to manage their daily activities [6]. Interestingly, those studies described similar patient concerns and goals despite being reported from different countries. We do not have enough knowledge, however, about the concerns, preferences and goals of "digital-age" younger women who have alternative information resources available on the internet, blogs and youtube [7]. This group is likely to have different information-seeking patterns and health awareness compared to the baby boomers who are our main patient population with pelvic floor disorders at present. On the other hand, the baby boomers are considered to be more proactive regarding their experience with pelvic floor disorders than the preceding generation of women as they were more conscious of youth, beauty and general appearance and wished to postpone aging [8]. As longevity increases, younger women will seek care for pelvic floor disorders, request more information about management options and discuss the available therapeutic interventions and will live long enough to experience treatment recurrences. We must be prepared to cope with this new task.

Current urogynecological practice is changing to respond to patient demands for scientific information about their symptoms, possible diagnoses and treatment options. In fact, most women with pelvic floor disorders will accept surgical intervention and welcome comprehensive clinical assessment provided that they understand the underlying pathology and receive explanation of the available treatment modalities [6]. Patient decision aids (PDA) are a very useful tool in this 
context [9]. Given that the information on the internet and in blogs can be confusing and contradictory, PDAs like fact boxes may help all women with pelvic floor disorders whether they are baby boomers or younger women in their decision making process. Data on success rates and surgical factors like hospital stay, postoperative complications, pain relief, type of anesthesia and recovery period can easily be summarized to the patient [9]. Ideally, the data should be individualized and based on the care provider's records and experience. The question "What would you recommend if I were your grandmother/mother /sister/daughter?" is consistently asked by many women with pelvic floor disorders when the offered treatment options are perceived to have the same outcome. Giving reasonable answers can be difficult but the provider must maintain a convincing knowledgeable attitude as well as a compassionate information-sharing approach that may include discussion of treatment-independent factors such as patient waiting lists, health insurance coverage and health care cost incurred.

In conclusion, Urogynaecology is a great but demanding subspecialty that requires outstanding communication skills. We should be proud that our treatment outcome research focuses not only on restoration of pelvic floor function but also on patient-centered measures. With the numerous and effective management options available for pelvic floor disorders, including suburethral tapes and vaginal mesh in many countries, women do and must have a choice in their treatment management. We have achieved great milestones in counseling women who use the traditional knowledge methods of health care. However, understanding the perceptions, preferences and goals of women who are mainly influenced by electronic-based health information is equally important.

\section{References}

1. Toozs-Hobson P, Freeman R, Barber M, et al. An International Urogynecological Association (IUGA)/International Continence Society (ICS) joint report on the terminology for reporting outcomes of surgical procedures for pelvic organ prolapse. Int Urogynecol J. 2012;23:527-35.

2. Raasthoj I, Elnegaard S, Rosendal M, Jarbol DE. Urinary incontinence among women-which personal and professional relations are involved? A population-based study. Int Urogynecol J. 2019;30: 1565-74.

3. Tinetti A, Weir N, Tangyotkajohn U, Jacques A, Thompson J, Briffa $\mathrm{K}$. Help-seeking behaviour for pelvic floor dysfunction in women over 55: drivers and barriers. Int Urogynecol J. 2018;29:1645-53.

4. Rizk DE, El-Safty MM. Female pelvic floor dysfunction in the Middle East: a tale of three factors-culture, religion and socialization of health role stereotypes. Int Urogynecol J. 2006;17:436-8.

5. Hoke TP, Berger AA, Pan CC, et al. Assessing patients' preferences for gender, age, and experience of their urogynecologic provider. Int Urogynecol J. 2019. https://doi.org/10.1007/s00192-019-04189-0.

6. Gray T, Strickland S, Pooranawattanakul S, et al. What are the concerns and goals of women attending a urogynaecology clinic? Content analysis of free-text data from an electronic pelvic floor assessment questionnaire (ePAQ-PF). Int Urogynecol J. 2019;30: $33-41$.

7. Mazloomdoost D, Kanter G, Chan RC, et al. Social networking and Internet use among pelvic floor patients: a multicenter survey. Am J Obstet Gynecol. 2016;215(654):e651-4.

8. LeRouge CM, Tao D, Ohs J, Lach HW, Jupka K, Wray R. Challenges and Opportunities with Empowering Baby Boomers for Personal Health Information Management Using Consumer Health Information Technologies: an Ecological Perspective. AIMS Public Health. 2014;1:160-81.

9. Jha S, Duckett J. Utility of patient decision aids (PDA) in stress urinary incontinence surgery. Int Urogynecol J. 2019;30:1483-6.

Publisher's note Springer Nature remains neutral with regard to jurisdictional claims in published maps and institutional affiliations. 